\title{
The intracoronary injection of recombinant human prourokinase in emergency interventional therapy for high thrombus load during ST-segment elevation myocardial infarction: a retrospective cohort study
}

\author{
Zuo-An Qin', Xue-Lin Lu², Quan Zhou³, Li Luo1, Zhi-Xiang Zhan', Ning Guo1, Liang-Qing Ge1 \\ 'Department of Cardiology, The First People's Hospital of Changde City, Changde 415003, Hunan, China. \\ ${ }^{2}$ Department of Pathology, The First People's Hospital of Changde City, Changde 415003, Hunan, China. \\ ${ }^{3}$ Department of Science and Education, The First People's Hospital of Changde City, Changde 415003, Hunan, China.
}

Correspondence to: Liang-Qing Ge, Department of Cardiology, The First People's Hospital of Changde City, Changde 415003, Hunan, China. E-mail: geliangqing@163.com

How to cite this article: Qin ZA, Lu XL, Zhou Q, Luo L, Zhan ZX, Guo N, Ge LQ. The intracoronary injection of recombinant human prourokinase in emergency interventional therapy for high thrombus load during ST-segment elevation myocardial infarction: a retrospective cohort study. Vesse/ Plus 2020;4:2. http://dx.doi.org/10.20517/2574-1209.2019.22

\author{
Received: 6 Jul 2019 First Decision: 23 Sep 2019 Revised: 17 Dec 2019 Accepted: 15 Jan 2020 Published: 12 Feb 2020 \\ Science Editor: Cristiano Spadaccio Copy Editor: Jing-Wen Zhang Production Editor: Tian Zhang
}

\begin{abstract}
Aim: The clinical effect of the injection of recombinant human prourokinase to infarction-related arteries during percutaneous coronary intervention $(\mathrm{PCI})$ in ST-segment elevation myocardial infarction (STEMI) patients who have slow reflow/no reflow was investigated.
\end{abstract}

Methods: Data from STEMI patients who underwent emergency PCl at the Chest Pain Center of the First People's Hospital of Changde City from April 2017 to December 2018 were collected for analysis.

Results: Whether the ST segment had decreased by more than $50 \%$ at 90 min and whether the creatine kinase isoenzyme and cardiac troponin I had decreased by more than 50\% were investigated. There were no significant differences in the indexes of color Doppler echocardiography (left ventricular ejection fraction, left ventricular enddiastolic diameter, ventricular aneurysm, and ventricular thrombus) within seven days after surgery and in the ventricular tachycardia/ventricular fibrillation between the two groups $(P>0.05)$. There was no significant difference in major adverse cardiovascular event between the two groups within one month after surgery $(P>0.05)$, but, there were significant differences in the Thrombolysis in Myocardial Infarction (TIMI) classification, corrected TIMI frame count, creatine kinase peak value, and the third-degree atrioventricular block $(P<0.05)$.

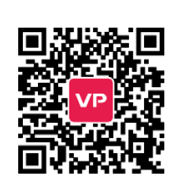


Conclusion: The intracoronary injection of recombinant human prourokinase in patients with STEMI undergoing emergency $\mathrm{PCl}$ is safe and effective.

Keywords: ST-segment elevation myocardial infarction, primary percutaneous coronary intervention, recombinant human prourokinase, infarction-related artery

\section{INTRODUCTION}

The guidelines of the European Society of Cardiology ${ }^{[1]}$ recommend percutaneous coronary intervention (PCI) as the preferred strategy for reperfusion therapy in patients with acute ST-segment elevation myocardial infarction (STEMI). However, when the thrombus load in the coronary artery is heavy, PCI cannot effectively remove the thrombus and increases the risk of thrombus shedding, leading to the occurrence of "slow blood flow" and "no reflow" in the distal infarction-related artery (IRA). For STEMI patients with a high coronary artery thrombus load, the treatment strategy is particularly critical, which has puzzled interventional doctors. The purpose of this study was to analyze the safety and efficacy of the intraoperative injection of recombinant human prourokinase (rhPro-UK) in STEMI patients with a high thrombus load during emergency PCI with IRA.

\section{METHODS}

\section{Patient enrollment}

From April 2017 to December 2018, 312 patients from the First People's Hospital of Changde City met the diagnostic criteria of STEMI based on the 2014 guidelines for the diagnosis and treatment of acute STEMI. All patients underwent emergency PCI, and the IRA was successfully opened. These patients were divided into two groups. Patients with high thrombus loads, which had been confirmed by coronary angiography, were treated with rhPro-UK by guiding catheter infusion into the proximal end of the IRA, and were placed in the Pro-UK group $(n=127)$. There were 109 males and 18 females in this group. The age of the Pro-UK patients ranged from 20 to 86 years old, and the average age was $59.56 \pm 12.58$ years old. Among these patients, 7 patients underwent percutaneous transluminal coronary angioplasty (PTCA) and 120 underwent PCI. Six people were treated with an intra-aortic balloon pump and 23 patients were treated with temporary pacemakers. For the affected vessels, there were 2 cases in the left trunk, 56 cases in the anterior descending branch, 8 cases in the circumflex branch, and 61 cases in the right coronary artery.

\section{Study protocol}

Pro-UK group: according to a high thrombus load, as confirmed by coronary angiography, the patients were treated with the intracoronary infusion of urokinase to the IRA. rhPro-UK was injected into the coronary artery, into a minimum of one branch, a maximum of nine branches ( $5 \mathrm{mg} / \mathrm{branch}$ ), and a median of two branches. Seven patients underwent PTCA and 120 underwent PCI.

Control group: in patients who did not have a high thrombus load during the operation, rhPro-UK was not injected into the coronary artery. Eight patients underwent PTCA and 177 underwent PCI.

Both groups were given aspirin $300 \mathrm{mg}$, clopidogrel 300/600 mg, or Ticagrelor $180 \mathrm{mg}$ immediately before the beginning of PTCA, followed by $100 \mathrm{mg} / \mathrm{qd}, 75 \mathrm{mg} / \mathrm{qd}$ or $90 \mathrm{mg} / \mathrm{bid}$, respectively.

\section{Statistical methods}

If measurement data had a normal distribution, the mean \pm standard deviation $(\mathrm{x} \pm \mathrm{s})$ are shown, and the difference between groups was compared by a $t$ test. If the data had a nonnormal distribution, they are represented by the median and quartile limits [M (Q1, Q3)], and the differences between these groups 
were compared by the Kruskal-Wallis rank sum test. The counting data are expressed as the percentage constituent ratio or rate, and the comparisons between groups were performed by the Chi-square test or Fisher test. Univariate and multivariate logistic regression analyses were used to identify the factors related to major adverse cardiovascular events (MACEs). All analyses were performed with the statistical software packages R (http://www.R-project.org, The R Foundation) and EmpowerStats (http://www.empowerstats. com, X \& Y Solutions, Inc, Boston, MA). $P$ values less than 0.05 (two-sided) were considered statistically significant.

\section{Observational indicators and methods}

Angiographic analysis

Coronary angiography was performed on the target lesion in the same projection to optimize the Thrombolysis in Myocardial Infarction (TIMI) blood flow classification of the IRA. A visual evaluation was performed by two experienced interventional cardiologists. The TIMI blood flow classification criteria were as follows: Grade 0 (no perfusion), no forward blood flow at the distal end of the vascular occlusion; Grade 1 (infiltration but no perfusion), the contrast medium was partially stopped and there was a plug site, but it did not fill distal blood vessels; Grade 2 (partial perfusion), the contrast medium could completely fill the distal end of the coronary artery, but the contrast medium filling and clearance speed was slow; and Grade 3 (complete perfusion), the contrast medium could completely and rapidly fill the distal blood vessels and was removed quickly.

\section{Biochemical assays and ST-segment resolution}

The concentrations of creatine kinase (CK) and creatine kinase isoenzyme (CK-MB) were measured by immunoassays (BECKMAN COULTER Au5800 instrument). Cardiac troponin I (cTnI) was measured by a fluorescence immunoassay (Mini VIDAS instrument). The peak levels of CK-MB and cTnI were used as indexes to judge the size of the infarction. An electrocardiogram (ECG) was recorded 90 min after the intervention, and a decrease rate of the ST segment of more than 50\% was regarded as the cutoff of myocardial reperfusion after PCI.

\section{Cardiac functions}

One week after emergency PCI, left ventricular ejection fraction (LVEF), left ventricular end-diastolic diameter (LVEDd), ventricular aneurysm, and ventricular thrombus were measured by Vivid 7 Dimension color Doppler echocardiography.

\section{Bleeding events and severe arrhythmias}

Severe postoperative bleeding events (gastrointestinal bleeding and cerebral hemorrhage) and severe postoperative arrhythmias [ventricular tachycardia/ventricular fibrillation (VT/VF) and third-degree atrioventricular block] were recorded.

\section{Follow-up}

The main MACEs within one month after operation were recorded.

\section{RESULTS}

\section{Baseline demographic, clinical, and angiographic characteristics}

There was no significant difference $(P>0.05)$ in sex, age, risk factors, Killip classification, IRA, time from onset to balloon dilatation, time from admission to balloon dilatation, number of dilated balloons used during surgery, number of stents implanted, intraoperative aortic balloon counterpulsation operations, and temporary pacemakers between the Pro-UK group and the control group [Table 1]. 
Table 1. Demographic, clinical, and angiographic characteristics of the subjects

\begin{tabular}{|c|c|c|c|}
\hline Variables & Pro-UK group $(n=127)$ & Control group $(n=185)$ & $P$-value \\
\hline Age (years) & $59.56 \pm 12.58$ & $61.34 \pm 11.73$ & 0.203 \\
\hline Male $[n(\%)]$ & $109(85.83 \%)$ & $146(78.92 \%)$ & 0.121 \\
\hline Hypertension $[n(\%)]$ & $70(55.12 \%)$ & $113(61.08 \%)$ & 0.293 \\
\hline Hyperlipemia $[n(\%)]$ & $34(26.77 \%)$ & $69(37.30 \%)$ & 0.052 \\
\hline Diabetes $[n(\%)]$ & $25(19.69 \%)$ & $39(21.08 \%)$ & 0.764 \\
\hline Smoking $[n(\%)]$ & $51(40.16 \%)$ & $65(35.14 \%)$ & 0.367 \\
\hline Killip $[n(\%)]$ & & & 0.259 \\
\hline I & $101(79.53 \%)$ & 149 (80.54\%) & \\
\hline II & $10(7.87 \%)$ & $23(12.43 \%)$ & \\
\hline III & $12(9.45 \%)$ & $10(5.41 \%)$ & \\
\hline IV & $4(3.15 \%)$ & $3(1.62 \%)$ & \\
\hline pre-PCI TIMI classification $[n(\%)]$ & & & $<0.001$ \\
\hline 0 & $107(84.25 \%)$ & $121(65.41 \%)$ & \\
\hline 1 & $14(11.02 \%)$ & $35(18.92 \%)$ & \\
\hline 2 & $6(4.72 \%)$ & $29(15.68 \%)$ & \\
\hline Time from symptom onset to balloon dilatation (h) & $6.00(4.50-9.00)$ & $7.00(5.00-10.00)$ & 0.193 \\
\hline Time from admission to balloon dilatation (min) & $65.00(48.50-85.00)$ & $64.00(47.00-93.00)$ & 0.202 \\
\hline Infarction-related artery $[n(\%)]$ & & & 0.083 \\
\hline LM & $2(1.57 \%)$ & $4(2.16 \%)$ & \\
\hline LAD & $56(44.09 \%)$ & $93(50.27 \%)$ & \\
\hline LCX & $8(6.30 \%)$ & $23(12.43 \%)$ & \\
\hline RCA & $61(48.03 \%)$ & $65(35.14 \%)$ & \\
\hline Number of implanted stents $[n(\%)]$ & & & 0.864 \\
\hline PTCA & $7(5.51 \%)$ & $8(4.32 \%)$ & \\
\hline 1 stent & $91(71.65 \%)$ & $134(72.43 \%)$ & \\
\hline 2 stent & $26(20.47 \%)$ & $36(19.46 \%)$ & \\
\hline 3 stents & $3(2.36 \%)$ & $7(3.78 \%)$ & \\
\hline Suction catheter $[n(\%)]$ & $15(11.81 \%)$ & $7(3.78 \%)$ & 0.007 \\
\hline IABP used $[n(\%)]$ & $6(4.72 \%)$ & $11(5.95 \%)$ & 0.640 \\
\hline Temporary pacemaker $[n(\%)]$ & $23(18.11 \%)$ & $27(14.59 \%)$ & 0.406 \\
\hline
\end{tabular}

LM: left main stem; LCX: left circumflex artery; RCA: right coronary artery; IABP: intra-aortic balloon pump; PTCA: percutaneous transluminal coronary angioplasty; $\mathrm{PCl}$ : percutaneous coronary intervention; TIMI: thrombolysis in myocardial infarction; Pro-UK: prourokinase; LAD: left anterior descending branch

\section{Evaluation of clinical effectiveness}

The following indexes of the two groups were analyzed: the TIMI classification of the IRA immediately after surgery and the corrected TIMI frame count (CTFC) were used to assess the blood perfusion of culprit vessels; whether the ST segment had decreased by more than $50 \%$ at $90 \mathrm{~min}$; and whether the peak of CK-MB and cTnI had decreased by more than 50\%. There were no significant differences in the indexes of color Doppler echocardiography (LVEF, LVEDd, ventricular aneurysm, and ventricular thrombus) within seven days after surgery $(P>0.05)$, but there was a significant difference in the TIMI classification, CTFC, and CK peak value $(P<0.05)$ between the two groups [Table 2].

\section{Clinical safety assessment}

Eight patients had gastrointestinal bleeding. Two patients experienced cerebral hemorrhage in the ProUK group, while 12 patients experienced gastrointestinal bleeding in the control group. There was no significant difference in the severity of complications $(P>0.05)$ or the length of hospital stay $(P>0.05)$ [Table 3] between the two groups. There was no significant difference in the VT/VF between the two groups $(P>0.05)$, but there was a significant difference in the third-degree atrioventricular block (III $\left.{ }^{\circ} \mathrm{AVB}\right)$ $(P<0.05)$ between the two groups [Table 3].

\section{Follow-up results}

Cardiovascular Major Adverse Events (MACEs) is a clinically viable technique for accurate, rapid, and safe evaluation of myocardial perfusion. In this study, MACE events included stent thrombosis, angina 
Table 2. Markers of infarct size, myocardial reperfusion, and cardiac functions

\begin{tabular}{lllc}
\hline Variables & Pro-UK group $(\boldsymbol{n}=\mathbf{1 2 7})$ & Control group $(\boldsymbol{n}=185)$ & $\boldsymbol{P}$-value \\
\hline Postoperative TIMI classification $[n(\%)]$ & & & 0.041 \\
0 & $1(0.79 \%)$ & $2(1.08 \%)$ & \\
1 & $0(0.00 \%)$ & $1(0.54 \%)$ & $6(3.24 \%)$ \\
2 & $14(11.02 \%)$ & $176(95.14 \%)$ & 0.047 \\
3 & $112(88.19 \%)$ & $24.16+3.65$ & 0.032 \\
CTFC (mm) & $23.26 \pm 2.68$ & $2356.50(1203.25-3797.00)$ \\
CK peak value (U/L) & $2625.50(1548.75-4582.50)$ & $204.30(96.30-329.65)$ & 0.217 \\
CK-MB peak value (U/L) & $229.55(110.60-371.33)$ & $7.55(2.29-20.33)$ & 0.511 \\
CTnl peak value (U/L) & $9.53(1.70-25.00)$ & $139(75.14 \%)$ & 0.198 \\
ST-segment resolution (> 50\%) $[n(\%)]$ & $87(68.50 \%)$ & $47.43 \pm 5.26$ & 0.307 \\
LVEDd (mm) & $48.08 \pm 5.73$ & $58.88 \pm 9.03$ & 0.569 \\
LVEF $(\%)$ & $58.28 \pm 9.30$ & $10(5.41 \%)$ & 0.067 \\
Ventricular aneurysm $[n(\%)]$ & $14(11.02 \%)$ & $2(1.12 \%)$ & 0.769 \\
Ventricular thrombus $[n(\%)]$ & $1(0.79 \%)$ & & \\
\hline
\end{tabular}

CK: creatine kinase; CK-MB: creatine kinase isoenzyme-MB; LVEF: left ventricular ejection fraction; LVEDd: left ventricular end-diastolic diameter; Pro-UK: prourokinase; TIMI: thrombolysis in myocardial infarction; CTFC: corrected TIMI frame count; cTnl: cardiac troponin I

Table 3. Comparison of the indexes of severe bleeding events, severe arrhythmia events, and hospitalization days

\begin{tabular}{lccc}
\hline Variables & Pro-UK group $(\boldsymbol{n}=\mathbf{1 2 7})$ & Control group $(\boldsymbol{n}=\mathbf{1 8 5})$ & $\boldsymbol{P}$-value \\
\hline Bleeding events & & & 0.655 \\
No & $117(92.13 \%)$ & $172(92.97 \%)$ & \\
Gastrointestinal bleeding & $8(6.30 \%)$ & $12(6.49 \%)$ & \\
Cerebral hemorrhage & $2(1.57 \%)$ & $1(0.54 \%)$ & 0.067 \\
VT/VF $[n(\%)]$ & $14(11.02 \%)$ & $10(5.41 \%)$ & 0.040 \\
III ${ }^{\circ}$ AVB $[n(\%)]$ & $12(9.45 \%)$ & $7(3.78 \%)$ & 0.444 \\
Hospitalization days (days) & $10.00(8.00-12.00)$ & $9.00(7.00-11.00)$ & \\
\hline
\end{tabular}

VT/VF: ventricular tachycardia/ventricular fibrillation; III ${ }^{\circ}$ VB: third-degree atrioventricular block; Pro-UK: prourokinase

Table 4. Comparison of MACEs between the two groups within one month of surgery

\begin{tabular}{lccc}
\hline Variables & Pro-UK group $(\boldsymbol{n}=\mathbf{1 2 7})$ & Control group $(\boldsymbol{n}=\mathbf{1 8 5})$ & $\boldsymbol{P}$-value \\
\hline Stent thrombosis $[n(\%)]$ & $0(0.00 \%)$ & $1(0.54 \%)$ & 0.407 \\
Angina pectoris $[n(\%)]$ & $6(4.72 \%)$ & $4(2.16 \%)$ & 0.207 \\
Stent rethrombosis $[n(\%)]$ & $2(1.57 \%)$ & $1(0.54 \%)$ & 0.358 \\
Congestive heart failure $[n(\%)]$ & $32(25.20 \%)$ & $38(20.54 \%)$ & 0.333 \\
Cardiac death $[n(\%)]$ & $7(5.51 \%)$ & $9(4.86 \%)$ & 0.799 \\
\hline
\end{tabular}

MACEs: major adverse cardiovascular events; Pro-UK: prourokinase

pectoris, stent rethrombosis, congestive heart failure, and cardiac death. There was no significant difference in MACEs between the two groups within one month after surgery $(P>0.05$; see Table 4$)$.

\section{Logistic regression analysis}

The MACEs that occurred within one month after surgery in the two groups were used as study Y and whether to use rhPro-UK as $\mathrm{X}$, and three logistic regression models were constructed according to the different confounding factors. The results of the rough model (without adjusting for any confounding factors), the microadjustment model (adjusting for only age and sex), and the overall adjustment model (adjusting for sex, age, hypertension, hyperlipidemia, diabetes, smoking, number of stents implanted, number of balloons used, Killip, post-PCI TIMI classification, time from symptom onset to balloon dilatation, and time from admission to balloon dilatation) show that the use of rhPro-UK was not significantly associated with the risk of MACEs $(P>0.05$; see Table 5). 
Table 5. Logistic regression analysis

\begin{tabular}{llll}
\hline Exposure & \multicolumn{1}{c}{ Non-Adjusted } & \multicolumn{1}{c}{ Adjusted I } & \multicolumn{1}{c}{ Adjusted II } \\
\hline Pro-UK group & 1.0 & 1.0 & 1.0 \\
Control group & $0.77(0.47,1.27) 0.3036$ & $0.73(0.44,1.22) 0.2292$ & $0.79(0.44,1.44) 0.4475$ \\
\hline
\end{tabular}

Adjusted model I: adjusted for sex and age; adjusted model II: adjusted for sex, age, hypertension, hyperlipidemia, diabetes, smoking, number of stents implanted, number of balloons used, Killip, post-PCI TIMI classification, time from symptom onset to balloon dilatation, and time from admission to balloon dilatation. Pro-UK: prourokinase; PCl: percutaneous coronary intervention; TIMI: thrombolysis in myocardial infarction

\section{DISCUSSION}

Emergency PCI can open IRAs in a timely and effective manner and can improve clinical prognosis. However, studies have shown that ${ }^{[2]}$ approximately 30\%-50\% of STEMI patients do not achieve effective myocardial reperfusion after PCI. Kaul et al. ${ }^{[3]}$ pointed out that myocardial perfusion disorder is an independent predictor of poor prognosis after emergency PCI. Considering that the burden of coronary artery thrombosis in patients with STEMI is often heavy, the compression of a balloon or stent during emergency PCI may result in the fragmentation and shedding of thrombi. The microthrombi that comprise thrombus segments detach from unstable plaques and aggregate with platelets, resulting in slow blood flow/no reflow ${ }^{[4,5]}$.

As part of a new generation of thrombolytic drugs, rhPro-UK can selectively activate fibrin-binding fibronase, converting fibronase into fibrase, which can dissolve thrombi and reduce the thrombus burden. rhPro-UK can prevent the occurrence of slow blood flow/no reflow during emergency PCI and does not affect the coagulation function of the whole body. In recent years, the treatment of intracoronary thrombolysis in PCI has represented a new era for thrombolysis/PCI combination therapy. Sezer et al. ${ }^{[6]}$ found that the intracoronary injection of streptokinase immediately after direct PCI can effectively increase myocardial perfusion. A domestic study by Zhao et al. ${ }^{[7]}$ showed that rhPro-UK had a high effectiveness for opening IRAs, and the incidence of bleeding complications was low, thus rhPro-UK is a safe treatment method. In another study, Zhao et al. ${ }^{[8]}$ showed that mechanical thrombectomy combined with rhPro-UK thrombolysis presented a more favorable efficiency in the treatment of moderate to severe acute cerebral infarction than single treatment, and the occurrence of adverse effects was similar between the combination and single treatments. In two other recent basic studies in animal models ${ }^{[9,10]}$, the results of the studies proved that rhPro-UK promoted thrombolysis and recanalization (patency rate) and did not increase the risk of bleeding. The abovementioned studies confirmed that the use of rhProUK can effectively promote thrombolysis without increasing the risk of bleeding. Our study showed that there was no difference in postoperative gastrointestinal bleeding and bleeding events between the two groups, suggesting that it is safe to administer rhPro-UK to IRAs through guiding catheters. The results are consistent with the abovementioned research results. In a recent study ${ }^{[11]}$, in patients with STEMI complicated with a long delay in PCI, emergency PCI combined with rhPro-UK thrombolysis showed significantly better myocardial perfusion of IRAs than direct PCI. Geng et al ${ }^{[11]}$ showed that the intracoronary injection of rhPro-UK through a balloon catheter could effectively improve myocardial perfusion in patients with STEMI. Our results show that the postoperative blood flow TIMI classification in the Pro-UK group was significantly higher than that before operation. Although there was a significant difference in the postoperative TIMI classification between the two groups, as a result of the degree of the thrombus load in the Pro-UK group, there was no difference in the ST return rate, color sonography, and MACEs between the two groups, suggesting that the administration of rhPro-UK to IRAs through guiding catheters is effective, which is consistent with the abovementioned results.

Previous studies have shown that ${ }^{[12]}$ rhPro-UK can be injected into the coronary artery in a variety of ways, such as guiding catheters, microcatheters, suction catheters, and drug balloons. Microcatheters or suction catheters require the guide wire to be withdrawn before drug injection and for the guide wire to 
be reinserted during treatment, which increases the surgery time and the risk of complications; however, the new perfusion balloon is expensive, which limits its clinical application. It has been reported ${ }^{[13]}$ that this drug could be injected into the target vessel through the side hole of the balloon by using a modified balloon catheter, and the high local concentration of this drug was used to treat slow blood flow/no reflow. However, the operation including the abovementioned methods is relatively complex, requiring a more extensive operation and prolonging the operation time, thus this procedure is not suitable for use in emergency PCI at most chest pain centers. In this study, rhPro-UK was directly injected into the coronary artery through a guiding catheter. This operation process is simple and does not affect the operation flow. We aimed to adopt a simple and low-cost method. At present, in the process of promoting the construction of a nationwide "chest pain center"in China, this method has been shown to be suitable for most chest pain centers. Although the administration of a guiding catheter leads to the outflow of part of the rhProUK out of the guiding catheter, most of the drug can reach the middle and distal end of the affected vessel in the direction of the blood flow, achieving effective results. In this study, there was no difference in the time from the onset to balloon dilatation and from admission to balloon dilatation between the Pro-UK group and the control group, suggesting that the administration of drugs through the guiding catheter did not affect the operation time and procedure. Additionally, with the improvement of myocardial reperfusion, there was no significant difference in the results of cardiac color Doppler ultrasounds between the two groups, including LVEF, LVEDd, ventricular aneurysm, ventricular thrombus, and segmental wall motion abnormality. Reinstadler et al. ${ }^{[14]}$ found that the ST-segment return rate was an important index of myocardial perfusion after emergency PCI. There was no difference in the ECG ST-segment return rate between the two groups. Previous studies have shown that ${ }^{[15]}$ a higher Killip grade is associated with higher mortality. In our study, MACEs were positively correlated with the Killip grade, as higher Killip grades were associated with a higher incidence of MACEs, which is consistent with the results described above. MACEs are a new clinically feasible measure of the accurate, rapid, and safe evaluation of myocardial perfusion ${ }^{[16]}$. During the follow-up period of one month, there was no difference in MACEs between the two groups. In this study, the ST return rate, based on ECG and color Doppler echocardiography, and MACEs were studied. There was no difference between the Pro-UK group and the control group in regard to these parameters. Multivariate regression analysis showed that there was no significant association between the use of rhPro-UK and the risk of MACEs. In conclusion, in conditions of high thrombus load during emergency PCI, rhPro-UK administration to the IRA through a guiding catheter was not associated with an increase in the incidence of bleeding complications and MACEs, suggesting that this method is safe and effective.

This study, in addition to having an insufficient sample size and a short follow-up time, was not a randomized controlled prospective study. Additionally, there was no standard dosage for all patients in the Pro-UK group. Considering that different dosages and routes of administration may affect the efficacy and safety of the drug, further study is needed to explore the optimal dose and route of administration of rhPro-UK in IRAs.

\section{DECLARATIONS}

\section{Authors' contributions}

Conceived the study, collected the data, participated in the design and drafted the manuscript: Qin ZA, Lu $\mathrm{XL}$

Participated in the design, collected the data, performed statistical analysis and helped to draft the manuscript: Qin ZA, Zhou Q, Lu XL

Helped to perform statistical analysis and to revise it critically for important intellectual content: Qin ZA, Luo L, Zhan ZX, Guo N, Ge LQ

All authors read and approved the final manuscript. 


\section{Availability of data and materials}

Not applicable.

\section{Financial support and sponsorship}

None.

\section{Conflicts of interest}

All authors declared that there are no conflicts of interest.

\section{Ethical approval and consent to participate}

Considering the retrospective nature of this study and the widely accepted use of rhPro-UK in China, specific consent form was waived.

\section{Consent for publication}

Not applicable.

\section{Copyright}

(C) The Author(s) 2020.

\section{REFERENCES}

1. Windecker S, Kolh P, Alfonso F, Collet JP, Cremer J, et al. 2014 ESC/EACTS guidelines on myocardial revascularization. Rev Esp Cardiol (Engl Ed) 2015;68:144.

2. Park SD, Baek YS, Lee MJ, Kwon SW, Shin SH, et al. Comprehensive assessment of microcirculation after primary percutaneous intervention in ST-segment elevation myocardial infarction: insight from thermodilution-derived index of microcirculatory resistance and coronary flow reserve. Coron Artery Dis 2016;27:34-9.

3. Kaul S. The "no reflow" phenomenon following acute myocardial infarction: mechanisms and treatment options. J Cardiol 2014;64:77-85.

4. Kodama T, Kondo T, Oida A, Fujimoto S, Narula J. Computed tomographic angiography-verified plaque characteristics and slowflow phenomenon during percutaneous coronary intervention. JACC Cardiovasc Interv 2012;5:636-43.

5. Schwartz BG, Kloner RA. Coronary no reflow. J Mol Cell Cardiol 2012;52:873-82.

6. Sezer M, Cimen A, Aslanger E, Elitok A, Umman B, et al. Effect of intracoronary streptokinase administered immediately after primary percutaneous coronary intervention on long-term left ventricular infarct size, volumes, and function. J Am Coll Cardiol 2019;54:1065-71.

7. Zhao L, Zhao Z, Chen X, Li J, Liu J, et al; Group of Prourokinase Phase IV Clinical Trials Investigators. Safety and efficacy of prourokinase injection in patients with ST-elevation myocardial infarction: phase IV clinical trials of the prourokinase phase study. Heart Vessels 2018;33:507-12.

8. Zhao QS, Li W, Li D, Liu T, Wang JH, et al. Clinical treatment efficiency of mechanical thrombectomy combined with rhPro-UK thrombolysis for acute moderate/severe cerebral infarction. Eur Rev Med Pharmacol Sci 2018;22:5740-6.

9. Hao CH, Ding WX, Sun Q, Li XX, Wang WT, et al. Effect of human recombinant prourokinase (rhpro-UK) on thromboembolic stroke in rats. Eur J Pharmacol 2018;818:429-34.

10. Hao C, Ding W, Xu X, Sun Q, Li X, et al. Effect of recombinant human prourokinase on thrombolysis in a rabbit model of thromboembolic stroke. Biomed Rep 2018;8:77-84.

11. Geng W, Zhang Q, Liu J, Tian X, Zhen L, et al. A randomized study of prourokinase during primary percutaneous coronary intervention in acute ST-segment elevation myocardial infarction. J Interv Cardiol 2018;31:136-43.

12. Han YL, Liu JN, Jing QM, Ma YY, Jiang TM, et al. The efficacy and safety of pharmacoinvasive therapy with prourokinase for acute ST-segment elevation myocardial infarction patients with expected long percutaneous coronary intervention-related delay. Cardiovasc Ther 2018;31:285-90.

13. Ettenson DS, Edelman ER. Local drug delivery: an emerging approach in the treatment of restenosis. Vasc Med 2000;5:97-102.

14. Reinstadler SJ, Baum A, Rommel KP, Eitel C, Desch S, et al. ST-segment depression resolution predicts infarct size and reperfusion injury in ST-elevation myocardial infarction. Heart 2015;101:1819-25.

15. Taguchi E, Konami Y, Inoue M, Suzuyama H, Kodama K, et al. Impact of Killip classifcation on acute myocardial infarction: data from the SAIKUMA registry. Heart Vessels 2017;32:1439-47.

16. Dijkmans PA, Senior R, Becher H, Porter TR, Wei K, et al. Myocardial contrast echocardiography evolving as a clinically feasible technique for accurate, rapid, and safe assessment of myocardial perfusion: the evidence so far. J Am Coll Cardiol 2016;48:2168-77. 